ARTICLE

https://doi.org/10.1038/s41467-019-08785-y

\title{
Divergent roles of FT-like 9 in flowering transition under different day lengths in Brachypodium distachyon
}

Zhengrui Qin ${ }^{1}$, Yuxue Bai ${ }^{1}$, Sajid Muhammad ${ }^{1}, \mathrm{Xia}_{\mathrm{W}}{ }^{1}$, Pingchuan Deng${ }^{1}$, Jiajie $\mathrm{Wu}^{2}$, Hailong $\mathrm{An}^{2} \&$ Liang Wu (iD ${ }^{1}$

Timing of reproductive transition is precisely modulated by environmental cues in flowering plants. Facultative long-day plants, including Arabidopsis and temperate grasses, trigger rapid flowering in long-day conditions (LDs) and delay flowering under short-day conditions (SDs). Here, we characterize a SD-induced FLOWERING LOCUS T ortholog, FT-like 9 (FTL9), that promotes flowering in SDs but inhibits flowering in LDs in Brachypodium distachyon. Mechanistically, like photoperiod-inductive FT1, FTL9 can interact with FD1 to form a flowering activation complex (FAC), but the floral initiation efficiency of FTL9-FAC is much lower than that of FT1-FAC, thereby resulting in a positive role for FTL9 in promoting floral transition when FT1 is not expressed, but a dominant-negative role when FT1 accumulates significantly. We also find that CONSTANS 1 (CO1) can suppress FTL9 in addition to stimulate FT1 to enhance accelerated flowering under LDs. Our findings on the antagonistic functions of FTL9 under different day-length environments will contribute to understanding the multifaceted roles of FT in fine-tune modulation of photoperiodic flowering in plants.

\footnotetext{
${ }^{1}$ College of Agriculture and Biotechnology, Zhejiang University, 310058 Hangzhou, China. ${ }^{2}$ State Key Laboratory of Crop Biology, Shandong Agricultural University, 271018 Tai'an, China. These authors contributed equally: Zhengrui Qin, Yuxue Bai. Correspondence and requests for materials should be addressed to L.W. (email: liangwu@zju.edu.cn)
} 
T he correct flowering timing is a critical determinant of the adaptation to different environments in plants. Flower meristem genes would be timely induced by a flowering activation complex (FAC), constituted by FLOWERING LOCUS $\mathrm{T}$ (FT), a small phosphatidylethanolamine binding protein (PEBP), and FD, a bZIP transcription factor by an assistance of scaffold protein 14-3-3 $\mathrm{s}$ in the cell nucleus, when plants respond to favorable flowering environments ${ }^{1,2}$. The diversification of FTs is important for floral initiation in plants, as different FTs or splicing isoforms may play opposite roles in flowering onset ${ }^{3,4}$.

Monitoring day length is one of the most important environmental cue for plant decision of reproductive transition. Facultative long-day plants, including Arabidopsis thaliana, trigger rapid flowering in long-day conditions (LDs) while delay flowering under short-day conditions (SDs) ${ }^{5}$. Under LDs, FT mRNA is induced by a key transcriptional regulator, CONSTANS (CO), though a direct interaction or an indirect association with FT ciselements ${ }^{6-8}$. Although clock-regulated and light-dependent $\mathrm{CO}$ mRNA and protein accumulation in plants are conserved and crucial for sensing day-length changes to regulate $F T$, however, lineage-specific plants may evolve special factors to control FTs under different light conditions 9,10 . For example, a temperate grasses-specific microRNA, miR5200, have been recently illustrated in a photoperiodic control of two FTs (FT1 and FT2) through a mRNA degradation approach ${ }^{11-13}$.

Compared with LD-induced floral promotion that has been extensively explored, the nature of default floral induction under SDs in LD-plants remains enigmatic ${ }^{14}$. Flowering of LD-plants under SD conditions has been considered mainly through plant hormone gibberellin (GA) signaling, but how plants percept and deliver GA signaling to trigger floral onset are still controversial ${ }^{15}$. Thereby, identification of more modulators in non-inductive environments can help understanding the exact molecular pathway of flowering initiation in plants.

Wheat and barley, two temperate grasses, provide us major sources of food and energy worldwide. Photoperiod sensitivity is an important agronomic event of wheat crop. Genetic study reveals that the adaptation to photoperiod in barley and wheat is predominantly determined by two Quantitative Trait Loci, PHOTOPERIOD 1 and 2 (Ppd-1 and Ppd-2) ${ }^{16}$. Ppd-1, an ortholog of $A$. thaliana PSEUDO-RESPONSE REGULATOR protein in circadian signaling, plays a major role in ear emergence in LDs through activation of $F T 1$ transcription ${ }^{17}$. Ppd-2 was identified to produce flowering time variation in SDs over two decades ago, however, the gene encoded Ppd-2 has not been clearly confirmed to date ${ }^{18}$. A short-day induced FT, FT3, is located to the same region in the chromosome as $P p d-2$ in barley, thereby recently thought to be the candidate encoder of Ppd-2, but the biological manner of FT3 is still unknown ${ }^{19-21}$. There is a close homolog named FT5 within the FT3 clade in barley, but its expression pattern and physiological involvement have not been determined to date as well ${ }^{19,22}$. Thereby, further molecular characterization of FT3 and FT5 is important to breed ideal genotypes for wider cultivation, using different combinations of their alleles in wheat and barley.

Brachypodium distachyon is an ideal model temperate grass for cereal flowering machinery investigation, due to its simple growth condition that can be artificially controlled with light and temperature $^{23}$. B. distachyon harbors all orthologs of the three core flowering proteins VERNALIZATION 1 (VRN1), VRN2, and FT1 in barley and wheat ${ }^{24}$. As those in barley and wheat, FT1 and VRN1 are behaved as promoters of flowering by forming a positive feedback loop in B. distachyon ${ }^{11,24,25}$. However, VRN2 in $B$. distachyon has been found induced during the vernalization process, in contrast with that in wheat and barley, which is suppressed by a cold treatment ${ }^{26,27}$. FT2 is a close paralog of FT1 in the temperate grasses. Recently, overexpression of FT2 has been observed giving rise to a precocious flowering both in $B$. distachyon and barely ${ }^{28}$, suggesting a similar role of FT2 in timing of the transition in B. distachyon and other temperate cereals. Accordingly, these results indicate that the orthologs of flowering proteins may have either similar or distinct functions in $B$. distachyon, wheat and barley.

There are also two protein members (FTL9 and FTL10) in FT3 clade in B. distachyon. Unlike that of in barley, FTL9 in B. distachyon is not affiliated in the same phylogenetic subgroup as FTL10 (Supplementary Fig. 1a), implicating that FTL9 is not derived from a recent duplication of FTL10 in B. distachyon, and thus may evolve alternative roles in flowering control ${ }^{29}$. In this study, we characterized FTL9 in B. distachyon, and observed a striking flowering performance in FTL9 loss-of-function and gain-of function plants. Mechanistically, we found that FACs by FTL9 and by canonical FT (FT1) had different flowering inductive abilities, resulting in an opposite behavior of FTL9 in flowering control in different day lengths. Moreover, we revealed that CO1 could suppress FTL9 to promote flowering under inductive LD conditions. Taken together, we illustrate a photoperiodic flowering pathway that is mediated by FTL9 and CO1 in $B$. distachyon.

\section{Results}

FTL9 is highly accumulated under SDs. In contrast to two FTlike proteins in A. thaliana, there are $6 \mathrm{FT}$ orthologs in temperate grasses $^{3,20}$ (Supplementary Fig. 1a). To explore the photoperiodic responses of $F T$ orthologs in temperate grasses, we examined the expressions of all $F T$-like genes at Zeitgeber time 4 (ZT4) under SDs and LDs in B. distachyon. FT1, FT2, FTL10, and FT4 are highly expressed after dawn under LDs whereas repressed in SDs (Fig. 1a). FT6 is difficult to be detected at any day-length conditions, perhaps due to its low expression (Fig. 1a). FTL9 is the sole $F T$ that is highly accumulated in SDs, while dramatically repressed in LDs (Fig. 1a), reminiscent that of FT3 in barley and wheat (Supplementary Fig. 1b) ${ }^{19-21}$, indicating that FTL9 in $B$. distachyon is a specific short-day induced FT. Thus, we further investigated its biological significance and molecular basis in detail.

To further pursue the effects of SDs on FTL9 expression, we examined the dynamic changes of FTL9 when plants were shifted from different day-length environments. There was a significant reduction of FTL9 transcription after plants moved from SDs to LDs for 3 days, and almost disappeared after 14-days movement (Fig. 1b). However, when LD-grown plants were transferred to SDs for 7 days, more than 40-fold FTL9 could be induced compared with that of under consistent LDs (Fig. 1c). Since FT family genes usually have diurnal expression rhythms, we examined the oscillation of FT1 and FTL9 every $4 \mathrm{~h}$ during the day-night cycle. In line with our previous results ${ }^{11}, F T 1$ displayed an expression peak at $4 \mathrm{~h}$ before dawn when plants were grown under LDs, but fully repressed under SDs (Supplementary Fig. 2a). By contrast, FTL9 was expressed to the highest level at the end of dark when plants were grown in SDs, and it was significantly repressed at any time points when plants were grown in LDs (Supplementary Fig. 2b).

PHYTOCHROME C (PHYC) is the critical light sensor in temperate grasses, and a couple of key flowering genes including FT1 and CO1 are greatly attenuated in phyc-1 mutant in LD photoperiod (Supplementary Fig. 3) 30-32 $^{3}$. By contrast, we found that there was an increase of 300-fold FTL9 in phyc-1 mutant than in wild-type plants under LDs (Fig. 1d), but only a very mild increase under SDs (Fig. 1e), suggesting that FTL9 is strictly controlled by PHYC under inductive photoperiod. 
a

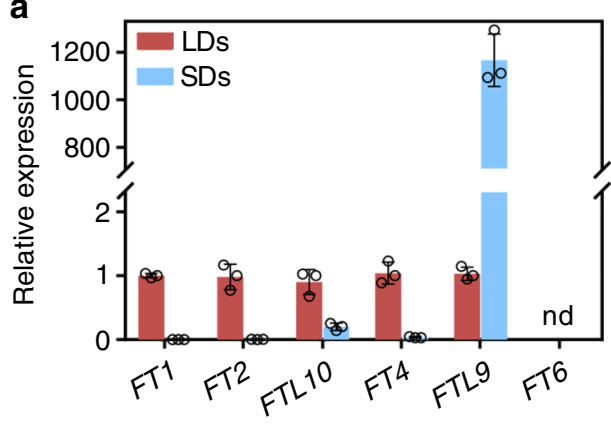

C

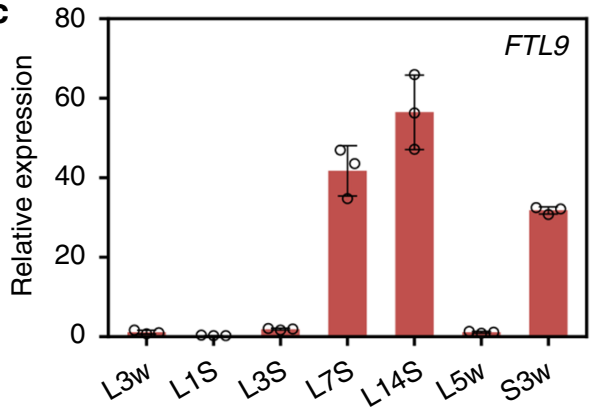

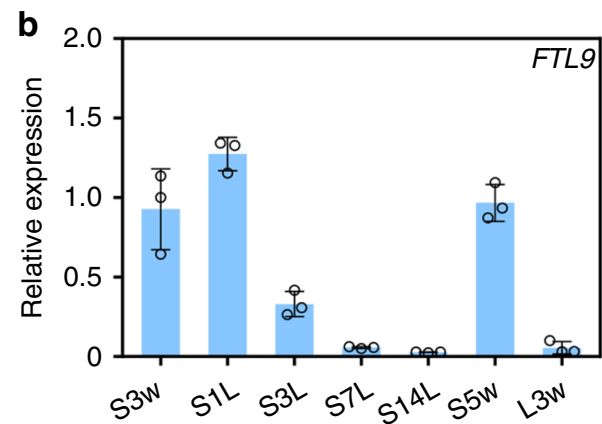
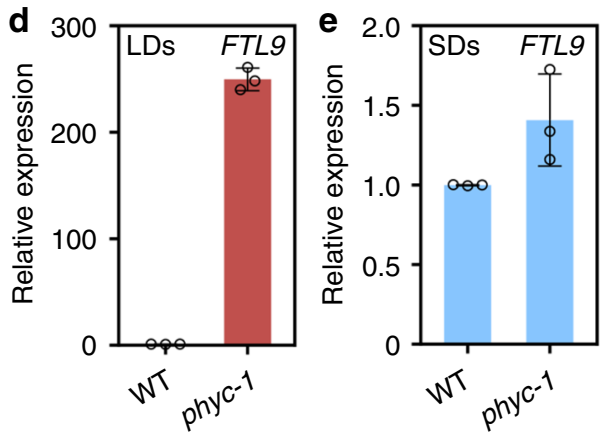

Fig. 1 FTL9 is specifically induced under SDs in B. distachyon. a qRT-PCR analysis of six FT orthologous gene expressions at ZT4 under SDs and LDs. UBC18 was used as an internal control for normalization of qRT-PCR results. Plant newly expanded leaves were harvested for RNA isolation and gene expression analysis. Error bars indicate s.d. b Time course analysis of FTL9 expression at ZT4 when three-week-old SD plants were shifted to LDs for 1 day (S1L), 3 days (S3L), 7 days (S7L), and 14 days (S14L), respectively. S3w and S5w indicate that $B$. distachyon were grown under consistent SDs for 3 and 5 weeks. $\mathrm{L} 3 \mathrm{w}$ indicates that $B$. distachyon were grown under LDs for 3 weeks as a control. UBC18 was used as an internal control for normalization of qRT-PCR results. Error bars represent s.d. c Time course analysis of FTL9 expression at ZT4 when three-week-old LD plants were shifted to SDs for 1 day (L1S), 3 days (L3S), 7 days (L7S) and 14 days (L14S), respectively. L3w and L5w indicate that B. distachyon were grown under consistent LDs for 3 and 5 weeks. S3w indicates that $B$. distachyon were grown under SDs for 3 weeks as a control. Error bars represent s.d. $\mathbf{d}$ qRT-PCR analysis of FTL9 expression under LDs in the wild-type Bd21-3 and phyc-1 homozyous mutants. UBC18 was used as an internal control for normalization of qRT-PCR results. Error bar means s.d. e qRT-PCR analysis of FTL9 expression under SDs in the wild-type Bd21-3 and phyc-1 homozyous mutants. Error bar means s.d.

Collectively, these results indicate a significant induction of FTL9 by short days in B. distachyon.

FTL9 is involved in flowering in SDs. FAC formation mediated by FT is essential for floral initiation. Our yeast two-hybrid, bimolecular fluorescence complementation (BiFC) and firefly luciferase complementation imaging (FLCI) assays show that FTL9 can associate with FD1 and 14-3-3 proteins to constitute a functional FAC as FT1, a canonical LD-induced florigen encoder (Fig. 2a-d and Supplementary Fig. 4) ${ }^{3}$.

Subsequently, we asked how FTL9-mediated FAC controls flowering in B. distachyon. To address this question, we generated FTL9 loss-of-function mutants through a CRISPR-cas9 approach (Fig. 2e). Since FTL9 is specially accumulated in SDs, we determined flowering time in two homozygous frame-shift mutants of ftl9 under SDs. We observed that they flowered almost 190 days after grown in soil under SDs, two month later than the wild-type (WT) plants (Fig. 2f, g). Together with the significant repressions of $V R N 1$ and FUL2, two flowering downstream genes, in $f t l 9$ mutants (Fig. $2 \mathrm{~h}$, i), these findings suggest that FTL9 is involved in flowering control under SD photoperiod.

FTL9-FAC has a mild flowering-inductive effect under SDs. To further explore the functions of FTL9 in SD-mediate flowering, we overproduced FTL9 in B. distachyon. FTL9 transcript level was 10 -fold to 20-fold increased in our several independent FTL9 overexpression (FTL9-OE) lines (Supplementary Fig. 5). As expected, the heading-date of FTL9-OE plants was significantly shortened under SDs (Fig. 2j, k), and VRN1 and FUL2 expressions were dramatically increased compared with WT (Fig. 2l, m), suggesting that FTL9 can indeed promote heading in $B$. distachyon under SDs. Nevertheless, it is notable that the reduction of flowering time in FTL9-OE is far from that in FT1 over-expression plants, which can lead to an extremely early flowering ${ }^{11,23-25}$, implying that although FTL9 is a positive regulator of flowering under SDs, its flowering inductive activity may not be dramatic. Interestingly, the expressions of FT1 are similar in FTL9-OE lines and wild-type plants (Supplementary Fig. 6), implicating that the induction of flowering under SDs by FTL9 is neither by a direct nor an indirect trigger of FT1.

We further investigated why FTL9 had such a mild flowering inductive effects under SDs. Since FAC in temperate grasses have been demonstrated with an ability to bind VRN1 cis-elements and promote VRN1 expression in wheat ${ }^{2,33}$, we determined the binding capacity of FTL9-FD1 and FT1-FD1 module to VRN1 promoter (VRN1p) when they simultaneously introduced into Nicotiana benthamiana leaf cells. As shown in Fig. 3a, the signal of luciferase (LUC) reporter triggered by FTL9-FAC-VRN1p was much lower than that by FT1-FAC-VRN1p, suggesting a finitely propulsive role of FTL9 in plant flowering.

To assess what makes the difference of flowering inductive capacity of FT1 and FTL9 in B. distachyon, we artificially swapped FTL9 and FT1 PEBP domain and transiently introduced them with FD1 into N. benthamiana leaf cells to compare the VRN1pLUC signal. We found that the VRN1p-LUC activation by FTL9 could be obviously enhanced when the FTL9 PEBP domain was changed to that of FT1, whereas VRN1p-LUC activation by FT1 
a

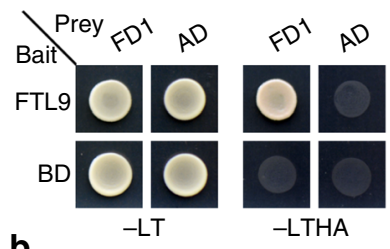

b

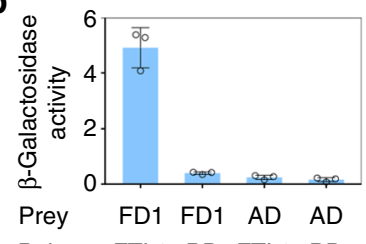

Bait FTL9 BD FTL9 BD
C

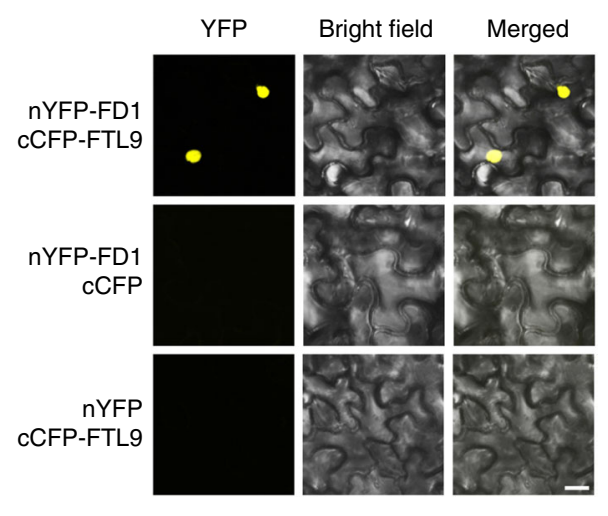

d

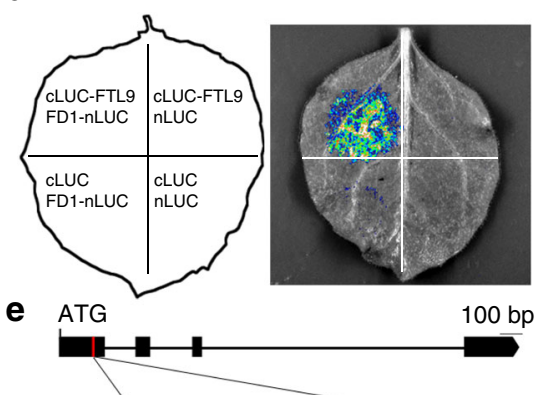

WT 5'-AACAAGCCAAGAGTTGATGTTGG-3'

ftla\#1 5'-AACAAGCCAAGAGTT--TGTTGG-3' -2

ftl|\$2 5'-AACAAGCCAAGA-----TGTTGG-3' -5
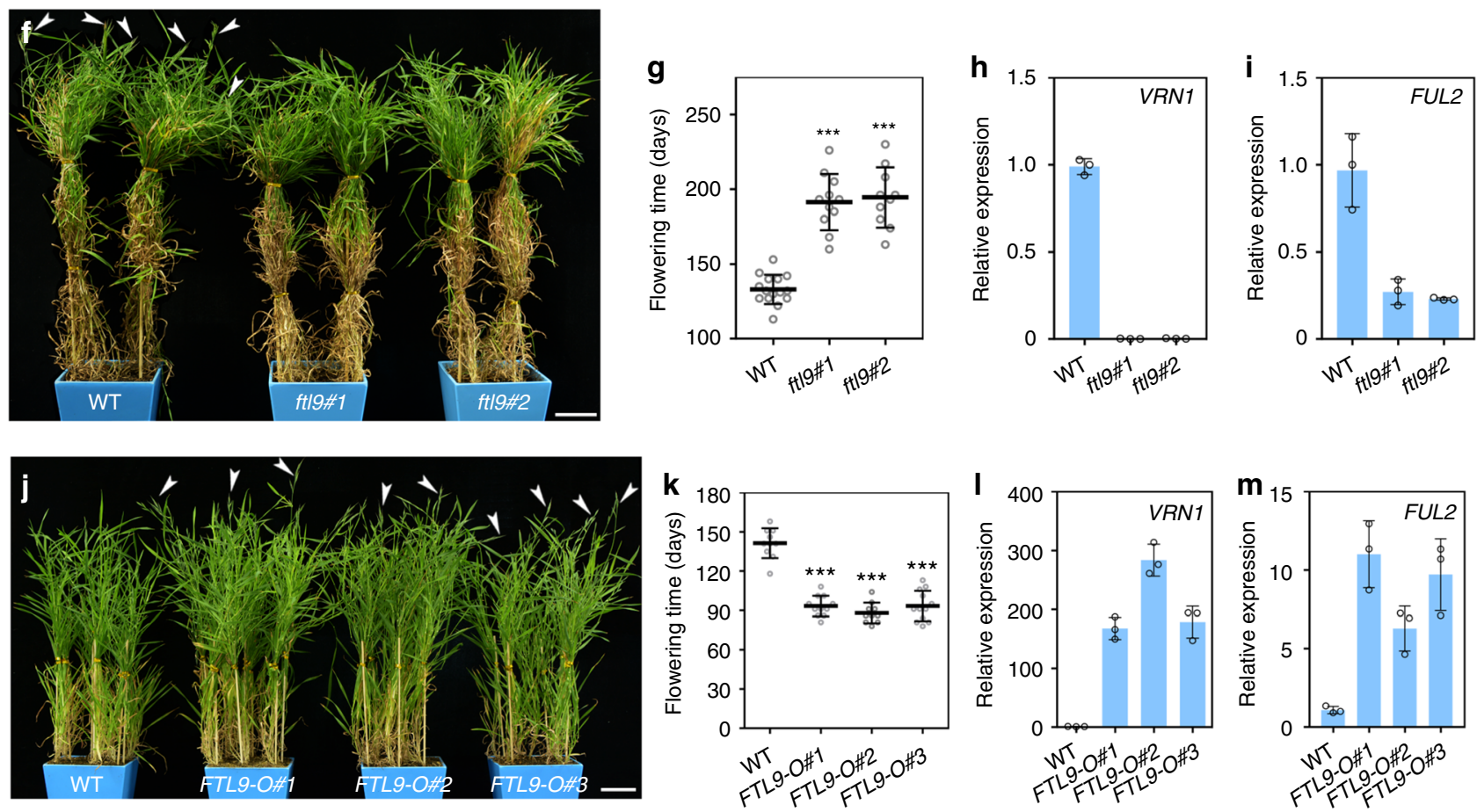

Fig. 2 FTL9 is a promoter of flowering under SDs in B. distachyon. a Interaction analysis between FTL9 and FD1 in yeast cells. Yeast cells growing on selective media without Leu, Trp, His and Ade (-LTHA) represent positive interactions. b Relative quantification of FTL9 and FD1 interactions by $\beta$-Gal activity in yeast two-hybrid assays. Bars indicate s.d. c BiFC assays for determination of FTL9 and FD1 interactions in N. benthamiana leaf cells. Scale bar, 20 $\mu \mathrm{m}$. d Firefly luciferase complementation imaging (FLCI) assays of FTL9 and FD1 interactions in N. benthamiana leaves. e CRISPR/Cas9-mediated target mutagenesis of FTL9. Top: a schematic diagram of the FTL9 bearing the CRISPR-Cas 9 target sites. Exons and introns are represented by black boxes and lines, respectively. The lower sequences show the alignment of wild-type Bd21, ft/9-1 and ft/9-2 sequences containing the CRISPR-Cas 9 target sites. The ft/9-1 and ft/9-2 mutants contain a 2-bp and 5-bp deletion, respectively. The black underlined sequences mean protospacer adjacent motifs.

f Representative phenotypes of two ft/9 mutants under SDs. White arrows point to spikes. Scale bar, $5 \mathrm{~cm}$. $\mathbf{g}$ Flowering time of wild-type (WT) Bd21 and the $f t / 9$ mutants under SDs. At least 10 plants for each line were scored. (Student's $t$ test, ${ }^{\star \star \star} P<0.001$ ). $\mathbf{h}$ qPCR analysis of flowering downstream gene VRN1 expression in eight-week-old wild-type and the indicated ft/9 mutants under SDs. Error bars indicate s.d. i qPCR analysis of flowering downstream gene FUL2 expression in eight-week-old wild-type and two ft/9 mutants under SDs. Error bars indicate s.d. $\mathbf{j}$ Representative phenotypes of three FTL9 overexpressing plants under SDs. White arrows point to spikes. Scale bar, $5 \mathrm{~cm}$. k Flowering time of wild-type Bd21 and the FTL9-OE plants under SDs. At least 10 plants for each line were scored. (Student's $t$ test, ${ }^{\star \star \star} P<0.001$ ). I qPCR analysis of flowering downstream gene VRN1 expression in eight-week-old wildtype and the FTL9-OE plants under SDs. Error bars indicate s.d. $\mathbf{m}$ qPCR analysis of flowering downstream gene FUL2 expression in eight-week-old wildtype and the FTL9-OE plants under SDs. Error bars indicate s.d.

could be significantly compromised when the FT1 PEBP domain was changed to that of FTL9 (Fig. 3b, c), suggesting that the distinction of PEBP domain of FTL9 and FT1 is an important determinant of their divergent flowering initiation activity.

Subsequently, we attempted to explore which amino acid confers the specific flowering activity of FTL9 compared with FT1, and thereby compared the proteins of FTL9 with other FTs that are known with a high flowering initiation activity in long- day plant $B$. distachyon and A. thaliana, including BdFT1, BdFT2, BdFTL10, AtFT and At TWIN SISTER OF FT (AtTSF) (Supplementary Fig. 7) $)^{11,25,34,35}$.We assumed that the specific amino acids of FTL9 at PEBP domain that are completely different with those in other FTs might be responsible for the FTL9 mild flowering activity (Supplementary Fig. 7). However, when we swapped Lysine (Lys, K) at residue 128, the sole amino acid that is entirely distinct from that in other FTs at FTL9 PEBP 
a

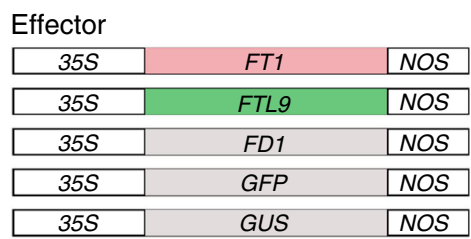

Reporter \begin{tabular}{|l|l|l|l|l|}
\hline VRN1P & LUC & $35 S$ & REN & NOS \\
\hline
\end{tabular}

\section{b}

Effector

\begin{tabular}{|c|c|c|}
\hline $35 S$ & FT1 & NOS \\
\hline $35 S$ & FTL9 & NOS \\
\hline $35 S$ & FTL9:FT1PEBP & NOS \\
\hline $35 S$ & FD1 & NOS \\
\hline $35 S$ & GFP & NOS \\
\hline
\end{tabular}

Reporter

\begin{tabular}{|l|l|l|l|l|}
\hline VRN1P & LUC & $35 S$ & REN & NOS \\
\hline
\end{tabular}
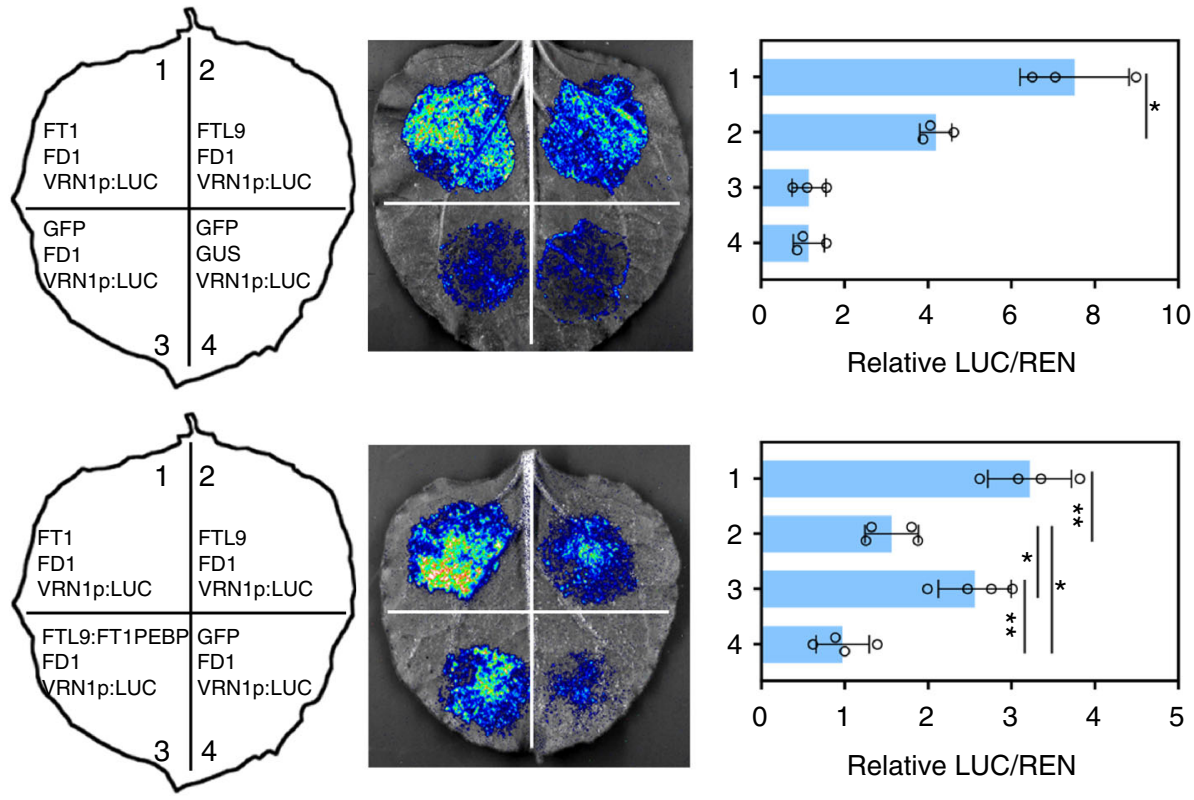

C

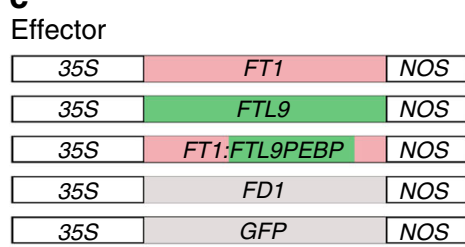

Reporter

\begin{tabular}{|l|l|l|l|l|}
\hline VRN1P & LUC & $35 S$ & REN & NOS \\
\hline
\end{tabular}
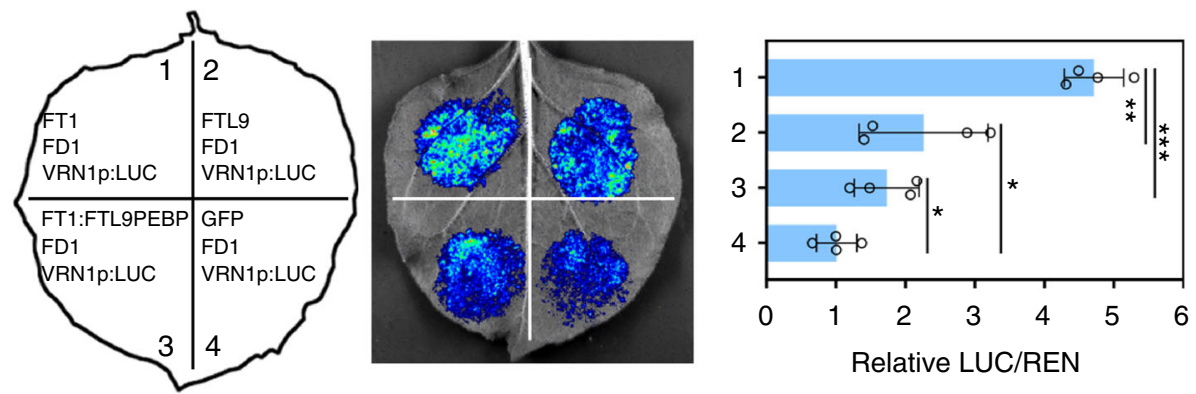

d

Effector
\begin{tabular}{|c|c|c|}
\hline $35 S$ & FT1 & NOS \\
\hline $35 S$ & FTL9 & NOS \\
\hline $35 S$ & FTL9-K128R & NOS \\
\hline $35 S$ & FD1 & NOS \\
\hline $35 S$ & GFP & NOS \\
\hline
\end{tabular}

Reporter

\begin{tabular}{|l|l|l|l|l|}
\hline VRN1P & LUC & $35 S$ & REN & NOS \\
\hline
\end{tabular}
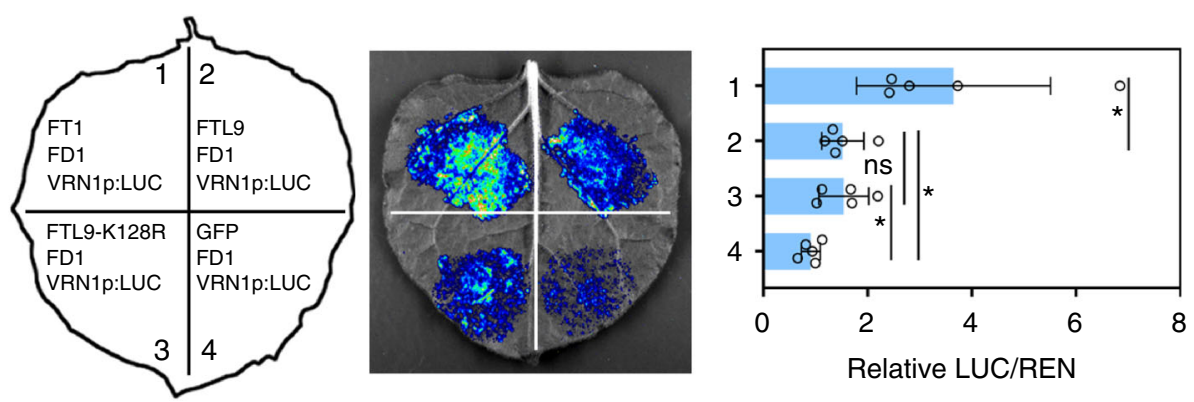

Fig. 3 FTL9-FAC has a weak flowering inductivity in B. distachyon. a The flowering inductive efficiency of FTL9-FAC is lower than that of FT1-FAC. Error bars indicate s.d. from different injected leaves. $\mathbf{b}$ FAC activity by FTL9 can be enhanced when the FTL9 PEBP domain is changed to that of FT1. PEBP domain of FTL9 is artificially swapped to that of FT1 and introduced to A.tumefaciens with FD1 and 3 kb VRN1 promoter-mediated LUC constructs, and co-infiltrated into $N$. benthamiana leaves. Error bars indicate s.d. c FAC activity by FT1 can be compromised when the FT1 PEBP domain is changed to that of FTL9. PEBP domain of FT1 is artificially swapped to that of FTL9 and introduced to A.tumefaciens with FD1 and 3 kb VRN1 promoter-mediated LUC constructs, and coinfiltrated into $N$. benthamiana leaves. Error bars indicate s.d. d Lysine at residue 128 of FTL9 is not responsible for the specific flowering ability of FTL9 in $B$. distachyon. Error bars indicate SD. (Student's $t$ test, ${ }^{\star} P<0.05,{ }^{\star \star} P<0.01,{ }^{\star \star \star} P<0.001$ ) Left: Different combinations of effectors and the reporter transiently introduced in N. benthamiana leaves. Middle: Representative photograph of firefly luciferase fluorescence signals when the indicated reporter and effectors were introduced in N. benthamiana leaves. Right: Relative reporter activity (LUC/REN) in N. benthamiana leaves expressing the indicated reporters and effectors

domain, to Arginine (Arg, R), we did not observe a conspicuous alteration of VRN1p-LUC signal compared with the original FTL9 (Fig. 3d), suggesting that only this Lys cannot be responsible for the specific flowering initiation capacity of FTL9, and whether a large fragment or other amino acids in FTL9 are the causes of its particular flowering action requires further determined.
Over-expression of FTL9 leads to a flowering delay under LDs. Because the flowering acceleration in FTL9-OE plants under SDs is not remarkable as that in canonical FT overproducing plants, we wondered what's their performance under LDs. Strikingly, we observed a significant delay rather than a precocity in heading in FTL9-OE plants (Fig. 4a, b), implicating that FTL9 may interfere plant flowering under LD conditions. Our further examination of 
VRN1 expression confirmed that over-expression of FTL9 can inhibit flowering under inductive photoperiod environments (Fig. 4c).

Next, we wondered why FTL9 has completely opposite actions in flowering control under different photoperiods. As we described above, FTL9 can interact with FD1 to form a functional FAC, but its flowering induction is not as efficient as FT1, thus it is possible that FTL9 can attenuate the FT1-FAC activity by competing with FT1 to FD1 when FT1 exists. To test this hypothesis, we transiently co-expressed FTL9, FT1, and FD1 in N. benthamiana leaves, and determined their effects on binding to $V R N 1$ promoter. As shown in Fig. 4d, the LUC signal triggered by the association between FT1-FAC and VRN1p is significantly compromised when FTL9 is introduced, however, there is no any decreases of LUC intensity when a control GFP or FT1 are coexpressed, suggesting that FTL9 is conferred with an inhibitive ability to FT1-FAC (Supplementary Fig. 8).

Taken together, the lower efficiency of FTL9-FAC than FT1FAC in floral initiation leads to a positive role of FTL9 in plant reproductive transition under SDs when FT1 is not expressed, while a dominant-negative effect when FT1 is significantly accumulated under LDs.

CO1 is a repressor of FTL9. CO has been shown as the central photoperiodic sensor that triggers flowering through activation of florigen genes in A. thaliana and many other plants ${ }^{36,37}$. Due to the contrary actions of FTL9 in flowering under different day lengths, we are curious whether FTL9 can be controlled by COlike proteins in B. distachyon.

There are two homologs of $\mathrm{CO}$ proteins in $B$. distachyon, termed $\mathrm{CO} 1$ and $\mathrm{CO} 2$, respectively (Supplementary Fig. 9a). To specifically knock down $\mathrm{CO} 1$ in $B$. distachyon, we chose the specific region of $\mathrm{CO} 1$ to produce double-strand RNA to silence $\mathrm{CO} 1$ in transgenic plants. As expected, $\mathrm{CO} 2$ expression is not decreased in CO1-RNAi lines (Supplementary Fig. 9b, c). Moreover, we generated CO1 over-expression lines (Supplementary Fig. 9d), and demonstrated that $\mathrm{CO} 1$ in B. distachyon could be functioning as a general CO-like protein, which promotes flowering by activating $F T 1$, and thereby increasing downstream VRN1 expression (Supplementary Figs. 10 and 11). Interestingly, when we measured the expression of FTL9 in CO1-RNAi plants under LDs, we found that FTL9 was obviously increased compared with WT (Fig. 5a). Furthermore, we observed a dramatic suppression of FTL9 in CO1-OE transgenic lines under SD conditions (Fig. 5b), suggesting that FTL9 is able to be repressed by $\mathrm{CO} 1$.

Consistent with genetic data, our molecular analysis found that the LUC signal triggered by FTL9 promoter in N. benthamiana leaves was pronouncedly decreased when we transiently introduced the CO1 protein (Fig. $5 \mathrm{c}$ ). These data indicates that CO1 regulates flowering in $B$. distachyon through FTL9 repression in addition to FT1 activation.

\section{Discussion}

Day length is a critical seasonal indicator that influences plant reproductive success. Current understanding of photoperiodic flowering in LD plants mostly be restricted to a long-day accumulated FT, which is temporal and spatial promoted by $\mathrm{CO}$, a oscillating sensor whose accumulation occurs at the right time of the day. This model nicely explains how flowering is induced under LDs, however, how plants trigger SD flowering is puzzled. In this study, we characterized a non-inductive short-day induced FT, FTL9, in B. distachyon (Fig. 5d). On the one hand, the mild flowering promotive activity of FTL9 is to ensure plant flowering eventually to propagate next generation under SDs; on the other hand, due to the weaker floral induction activity of FTL9-FAC compared with that of FT1-FAC, FTL9 becomes a dominatenegative repressor of flowering when FT1 is significantly accumulated under LDs.

The action of FTL9 is particular of biological significance, especially for winter varieties of temperate grasses, which must go through a short-day and low-temperature winter to confer flowering inductive ability. The loss-of-function of FTL9 can give rise to a delayed flowering under SD photoperiod, otherwise, the sensitive floral organs would be subject to a low-temperature damage if the plants are premature flowering in winter. When day length shifts to a long period in spring, winter plants would initiate flowering through a combinative approach, including activation of FT1 and decrease of FTL9. Thereby, our mechanistic elucidation of divergent roles of FTL9 under different photoperiods can provide some insights into creating proper cereal accessions in adaptation to broader agricultural environments and climate changes.

It is worthwhile to note that although $V R N 1$ is increased in FTL9-OE plants whereas decreased in ftl9 mutants, the acceleration of flowering by FTL9 in SDs may not be solely attributed to VRN1 activation, because it has been shown that over-expression of VRN1 does not result in a rapid flowering under $\mathrm{SDs}^{26}$. Besides, even VRN1 is higher in enhancer of zeste-like, a mutant of orthologous gene of A. thaliana CURLY LEAF 1, than wild-type plants under SDs, it still cannot trigger a more rapid flowering ${ }^{38}$. Therefore, expression of VRN1 may not be sufficient to induce flowering in B. distachyon under SD. Further identification of more FTL9 regulatory genes is valuable to illustrate the cause of reproductive transition in $B$. distachyon under non-inductive environments.

A recent study showed that most vernalization varieties of barley possessed the $p p d-H 2$ allele, a recessive allele of $F T 3^{39}$ There are two close homologs of FT3 in barley, named FT3 and FT5, and perhaps they are evolved from a gene duplicate event ${ }^{22}$. Although $B$. distachyon FTL9 and FTL10 belong to a big clade of FT3 proteins in temperate cereals, FTL9 in B. distachyon is not affiliated to the same subclade with FT3 in barley (Supplementary Fig. 1a). Because FT3 in barley accelerates the initiation of spikelet primordia independently of the photoperiod ${ }^{34}$, while FTL9 in $B$. distachyon has divergent roles in flowering transition under different day lengths, it would be interesting to know whether $B$. distachyon FTL10 has the same effect on flowering as FT3 in barley or FTL9 in B. distachyon. There is lack of B. distachyon FTL9 subclade proteins in barley and wheat, but in other grass species outside Tritaceae, such as rice, sorghum and maize, $B$. distachyon FTL9 has clear orthologs (Supplementary Fig. 12). It has been revealed that Zea mays CENTRORADIALIS 12 (ZCN12), a FTL9 ortholog in maize, can be induced by a SD treatment, but whether ZCN12 can function as $B$. distachyon FTL9 to compete with the floral activator, ZCN8, to postpone flowering under noninductive photoperiod is still unknown ${ }^{40,41}$. The FTL9 ortholog SbFT8 in sorghum when overexpressed in A. thaliana results in an extremely rapid flowering, and can rescue the flowering defects in A. thaliana ft mutants under LDs, implicating that FTL9 in $B$. distachyon and SbFT8 in sorghum may play different roles in activation of flowering ${ }^{42}$. Future work on exploring the biological relevance of FTL9 orthologs in short-day plants may be helpful to explain why $B$. distachyon harbors but wheat and barley lose FTL9 gene lineage.

As the central flowering pathway hub, FT family protein generally has consistent roles in regulating reproductive development, even though some FT homolog represses this transition $^{43}$. In a previous study, we demonstrated that one FT in temperate grass, FT2, can play opposite roles in floral onset through an alternative splicing strategy, which is simultaneously 

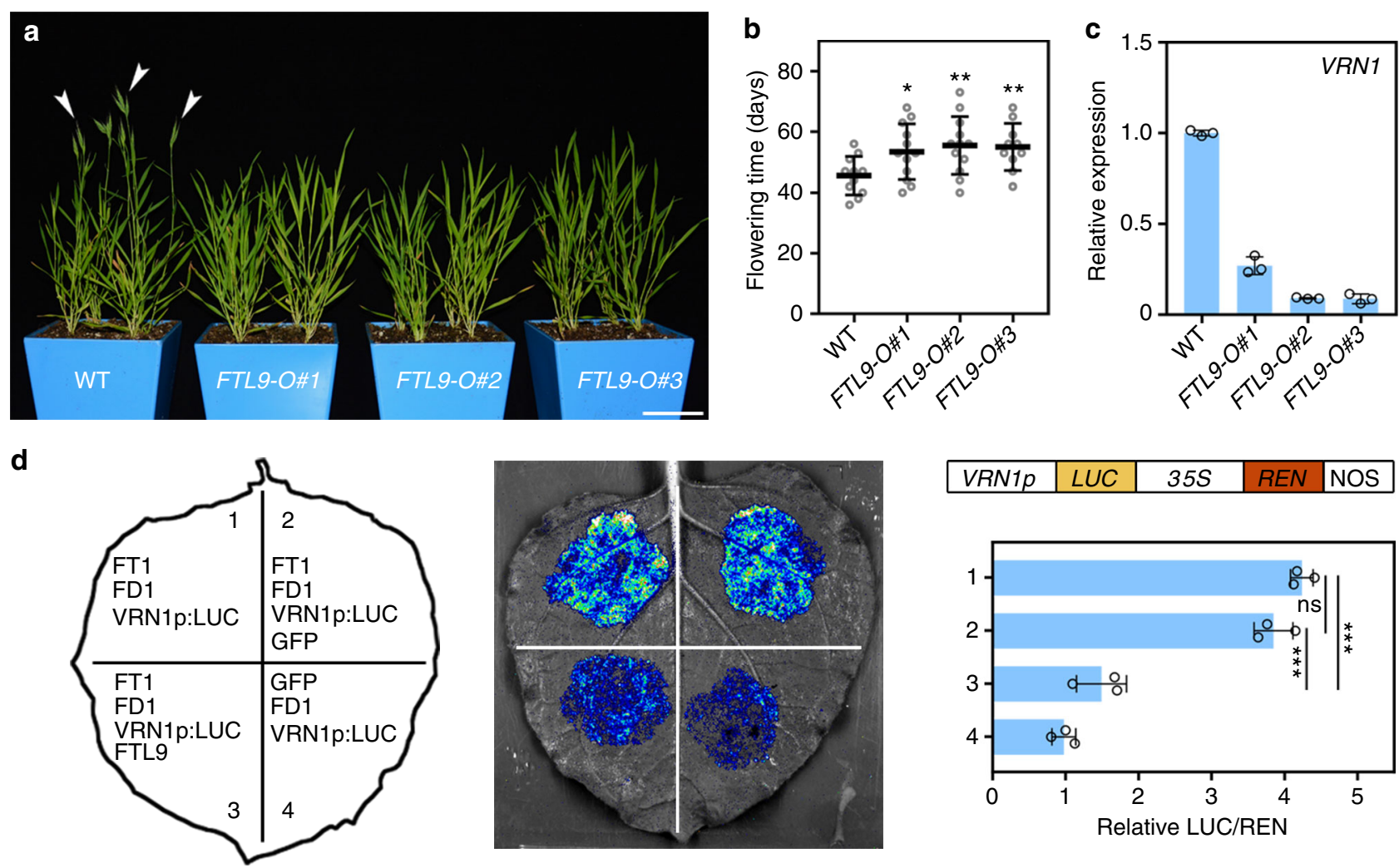

Fig. 4 Over-expression of FTL9 results in a flowering delay under LDs in B. distachyon. a Representative phenotypes of three FTL9 over-expressing lines under LDs. White arrows point to spikes. Scale bar, $5 \mathrm{~cm}$. b Flowering time of wild-type Bd21 and the FTL9-OE plants under LDs. At least 10 plants for each line were scored. (Student's $t$ test, ${ }^{\star \star} P<0.01,{ }^{\star} P<0.05$ ). c qPCR analysis of flowering downstream gene VRN1 expression in four-week-old wild-type and the FTL9-OE plants under LDs. Error bars indicate s.d. d FTL9 attenuates the binding ability of FT1-FAC to VRN1 cis-elements. Left: Different combinations of effectors and the reporter transiently introduced in $N$. benthamiana leaves. Middle: Representative photograph of firefly luciferase fluorescence signals

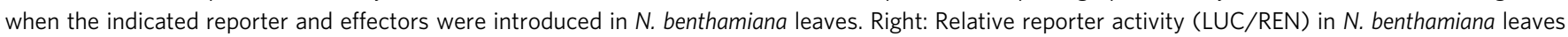
expressing the indicated reporters and effectors. Error bars indicate s.d. (Student's $t$ test, ${ }^{\star \star \star} P<0.001$ )

generating two isoforms that positively and negatively involved in age flowering pathway, respectively ${ }^{3}$. Together with our present finding that FTL9 has adverse manners in flowering control under different photoperiods, these results suggest that one FTlike protein can be conferred multifaceted roles in heading date in temperate grasses. Because there are more FT close orthologs in temperate grasses than $A$. thaliana, it is interesting to investigate whether other FT genes can also perform divergent roles in flowering transition.

In A. thaliana, $\mathrm{CO}$ is a direct activator of FT in the leaf vasculature to trigger flowering under facultative environments ${ }^{1,37}$. However, the CO ortholog in rice, Heading date 1 (Hd1), has dual characters in flowering control, accelerating flowering under SDs through promotion of the $\mathrm{Hd} 3 \mathrm{a}$, the FT ortholog, while suppressing flowering under $\mathrm{LDs}$ by repression of $\mathrm{Hd} 3 \mathrm{a}^{44,45}$. A recent finding explains the opposite regulatory activity of $\mathrm{Hd} 1$ in response to day length that is mediated by DAYS TO HEADING 8 , a NUCLEAR FACTOR Y (NF-Y) transcription factor that can physically interact with $\mathrm{Hd}_{1}^{46,47}$. Similar as that in rice, a $\mathrm{CO}$ ortholog, $\mathrm{CO} 1$, in $B$. distachyon can perform dual roles in triggering flowering under LDs, activating FT1 and inhibiting FTL9, although how $\mathrm{CO} 1$ can recognize different targets remains unknown. Since NF-Y is able to directly associate with or recruit histone modifiers on FT cis-elements ${ }^{6,48,49}$, we propose that CO1 in $B$. distachyon may interact with alternative NF-Y transcription factors to recognize different FT targets. Further studies are required to explore the specific module by CO1 and NF-Y to activate FT1 and repress FTL9 to trigger flowering under LDs in B. distachyon.

Meanwhile, although FTL9 could be regulated by CO1, the expression of FTL9 is not extremely high in CO1 knock-down lines under LDs. Given that there are two close $\mathrm{CO}$ orthologs in $B$. distachyon, $\mathrm{CO} 2$ may function redundantly with $\mathrm{CO} 1$ to suppress FTL9 to affect flowering. Supporting this hypothesis, we did not observe a remarkably precocious flowering in CO1-RNA $i$ lines under SDs compared with wild-type plants (Supplementary Fig. 13). Nonetheless, this also may be due to the non-functions of $\mathrm{CO} 1$ under SDs in $B$. distachyon, since $\mathrm{CO}$ protein has been shown to be degraded by a COP1-mediated ubiquitination complex in the evening in plants ${ }^{50-52}$.

It is also possible that FTL9 could be regulated by other modulators in addition to CO-like proteins. VRN2 is such a candidate because VRN2 represses $P P D-H 2$ has been shown in barley ${ }^{53,54}$. Additionally, VRN2 is expressed in LD but not in SD, and acts as a repressor of flowering in $B$. distachyon ${ }^{26,27,55}$. Lastly, VRN2 is also attenuated in the phyc-1 mutants and our CO1-RNAi transgenic lines (Supplementary Fig. 14a, b) ${ }^{30}$. Hence, VRN2 may mediate flowering control via suppression of FTL9 in B. distachyon. Intriguingly, our $\mathrm{CO} 1$ overexpressing $B$. distachyon lines can trigger a premature flowering, even if VRN2 is prominently enhanced compared with wild-type plants (Supplementary Fig. 14c). In barley, the effects on flowering of CO over-expression depends on VRN2, inducing flowering when VRN2 loses while repressing flowering when VRN2 exists ${ }^{36}$. Thus, CO and VRN2 

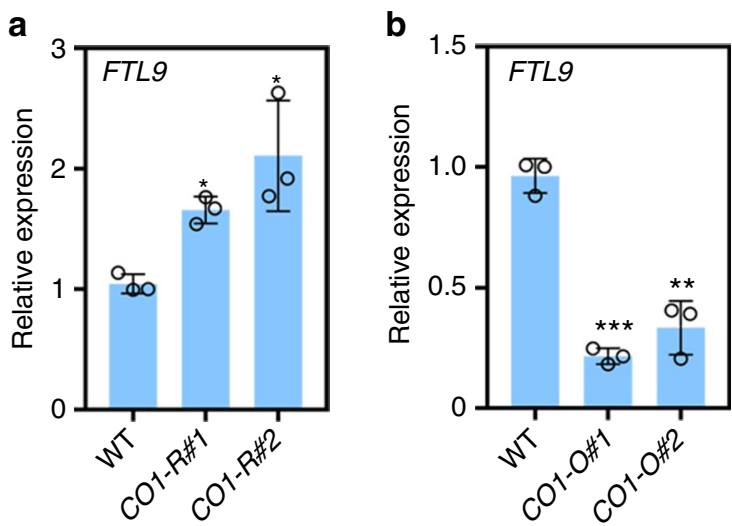

d

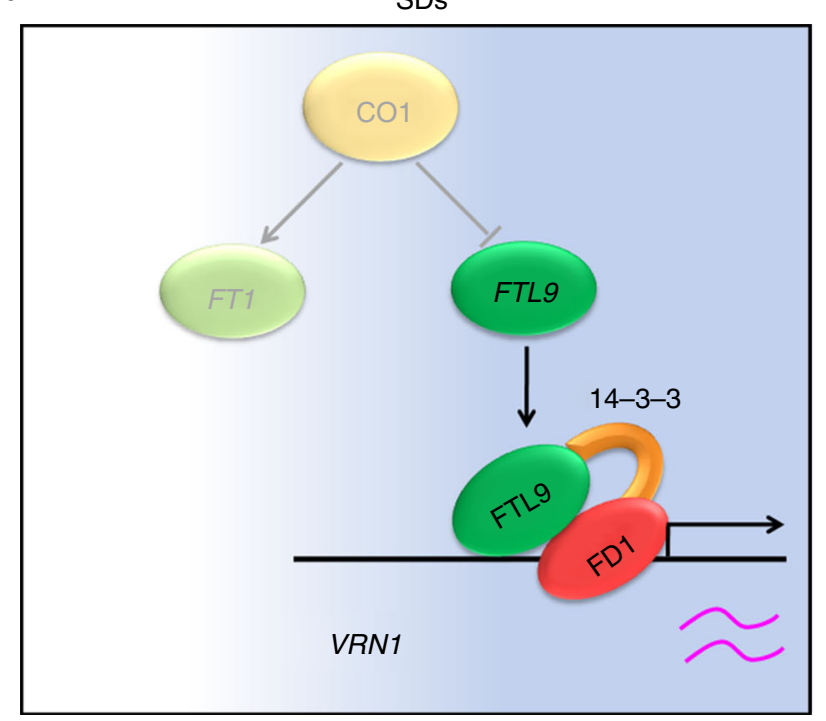

C

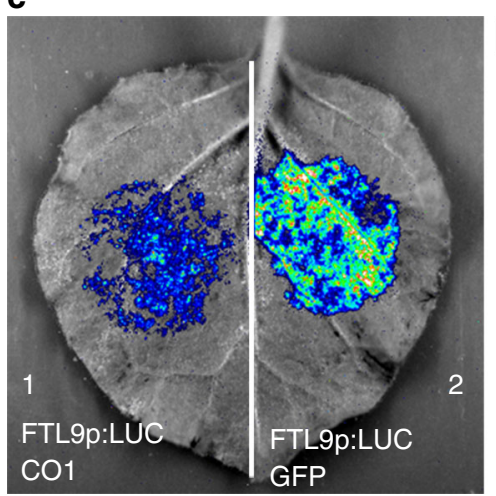

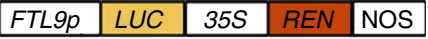

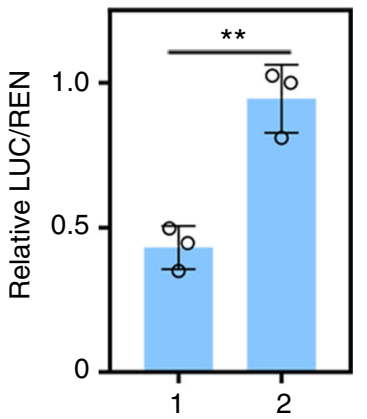

LDs

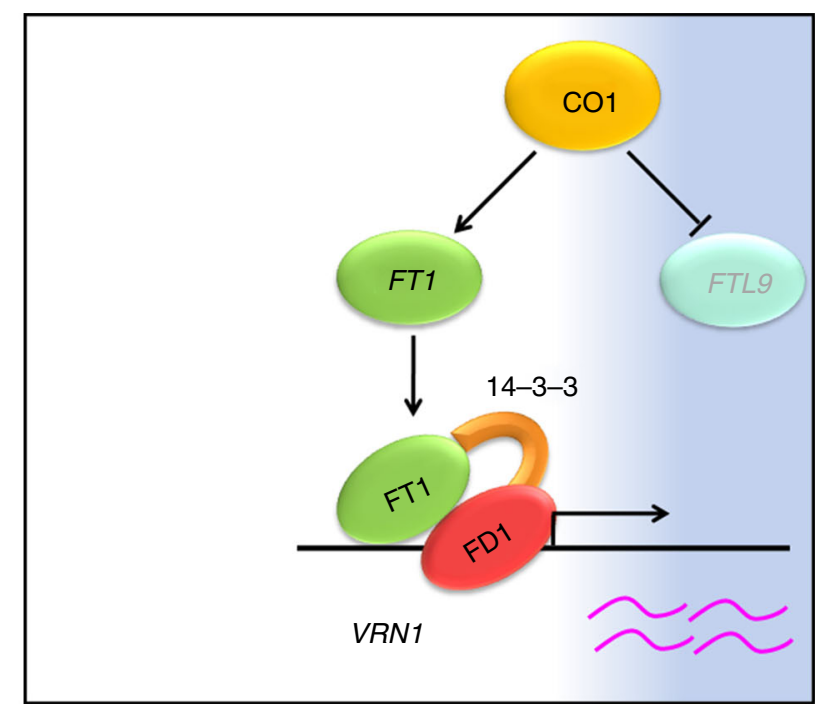

Fig. 5 CO1 negatively regulates FTL9 in B. distachyon. a qPCR analysis of FTL9 expression in four-week-old wild-type Bd21-3 and two independent CO1-RNAi $(C O 1-R)$ lines under LDs. Error bars indicate s.d. (Student's $t$ test, $\left.{ }^{\star} P<0.05\right)$. b qPCR analysis of $F T L 9$ expression in four-week-old wild-type Bd21-3 and two independent $\mathrm{CO} 1$ overexpressing (CO1-O) transgenic lines under SDs. Error bars indicate s.d. (Student's $t$ test, ${ }^{\star \star} P<0.01,{ }^{\star \star \star} P<0.001$ ). c Examination of $\mathrm{CO} 1$ repressive activity to FTL9 in N. benthamiana leaves. Left: Representative photograph of firefly luciferase fluorescence signals when the indicated reporter and effectors were introduced in N. benthamiana leaves. Right: Relative reporter activity (LUC/REN) in N. benthamiana leaves expressing the indicated reporters and effectors. Error bars indicate s.d. (Student's $t$ test, ${ }^{\star \star} P<0.01$ ). $\mathbf{d}$ A working model for the regulatory pathway of flowering initiation in B. distachyon under different day lengths. FTL9 can constitute a FAC with FD1 to trigger flowering, but the flowering inductive efficiency of FTL9-FAC is much lower than that of FT1-FAC, thereby resulting in a positive role of FTL9 in plant reproductive transition when FT1 is not expressed, while a dominantnegative role when FT1 is accumulated. The transcription and stabilization of $\mathrm{CO}$ is largely dependent on light in plants. In temperate grass, a CO ortholog, CO1, functions as a activator of FT1 whereas a repressor of FTL9. The decrease of CO1 mRNA and protein under SDs leads to an inhibition of FT1 and a release of FTL9, which makes plants a delay but an eventual floral onset in the non-inductive photoperiod. When plants grow under LDs, the accumulation of CO1 mRNA and protein is able to lead to a significant increase of FT1 and a dramatic reduction of FTL9, which triggers a rapid flowering

may have different interactions in barely and B. distachyon. Further research will be required to seek additional upstream regulators of FTL9 in the photoperiodic flowering pathway.

\section{Methods}

Plant growth and flowering time measurements. Brachypodium distachyon plants were sown in soil and grown with the temperature $22^{\circ} \mathrm{C} / 18^{\circ} \mathrm{C}$ days/nights and the approximate 12000 Lux light intensities. Flowering time was scored as the number of days from the date of planting in soil to the first day that emergence of the spike was detected. At least 10 plants of the transgenic line and the wild-type plants were used to score flowering time, which was measured in days. Unless stated otherwise, all plants in the study were B. distachyon $\mathrm{Bd} 21$ accession, grown in growth chambers under LD ( $16 \mathrm{~h}$ of light/ $8 \mathrm{~h}$ of dark) or SD (10 h of light/14 h of dark) conditions. The seedlings or leaves used in the experiments were generally harvested at Zeitgeber time 4.

Constructs and plant transformation. To generate UBI::FTL9, the full-length coding sequence (CDS) of FTL9 was amplified from B. distachyon Bd21 cDNA using primers shown in Supplementary Table 1, and cloned into the binary vector pCUBI1390 with a maize ubiquitin promoter. To construct FTL9 CRISPR-Cas9 system, the sgRNA expression cassette containing FTL9 target sequence under rice $\mathrm{U} 3$ promoter was generated by an overlapping PCR approach. Then, the PCR products and the binary vector pYLCRISPR/Cas $9 \mathrm{P}_{\mathrm{Ubi}^{-}}$ $\mathrm{H}$ were ligated in a mixture after simultaneously digested by BsaI (New England Biolabs). The ligated products with sgRNA expression cassettes were directly transformed into E. coli DH5a competent cells ${ }^{56}$. Chimeric FTL9:FT1 PEBP was constructed by replacing the residues $23-160$ of FTL9 with the residues 25-163 of FT1, and FT1:FTL9 PEBP was constructed by replacing the residues 25-163 of FT1 with the residues 23-160 of FTL9. The full-length CDS of CO1 was amplified from Bd21 B. distachyon cDNA and constructed into pCUBI1390 under a maize ubiquitin promoter to produce $\mathrm{CO} 1$ constitutively expressing vector. The $\mathrm{CO} 1$ gene specific region was obtained from $B$. distachyon cDNA, and cloned into the pANDA- $\beta$ vector to produce a hairpin structure of $\mathrm{CO} 1$ fragment for RNA silencing under maize ubiquitin promoter. All gene overexpression, RNAi and CRISPR/Cas 9 clones were validated by sequencing and then transformed into Agrobacterium tumefaciens AGL1 strain. B. distachyon transformation was based on the infection of embryogenic callus generated from immature embryos with A. tumefaciens followed by the selection of transgenic 
tissues on media with antibiotic hygromycin ${ }^{57}$. Independent transgenic lines were genotyped using HPT II-specific forward and reverse primers.

Gene expression analysis. Newly expanded leaves from at least six plants mixed for each indicated line were collected for RNA extraction and gene expression analysis. Total RNA was extracted using RNAiso Plus (Takara) and reversely transcribed using reverse transcriptase (Promega). cDNAs were generated from reverse transcription using Oligo (dT) primers ${ }^{3}$. Real-time PCR was done in triplicates on Step-One Plus real-time PCR system (ABI) with the Power Up SYBR Master Mix (ABI) with three biological replicates. The following cycling conditions were used for Real-time PCR: 2 min at $95^{\circ} \mathrm{C}, 40$ cycles of $10 \mathrm{~s}$ at $95^{\circ} \mathrm{C}$, and $40 \mathrm{~s}$ at $65^{\circ} \mathrm{C}$, and a final step for melting curve determination $\left(15 \mathrm{~s}\right.$ at $95^{\circ} \mathrm{C}, 1 \mathrm{~min}$ at $60^{\circ} \mathrm{C}$, and $15 \mathrm{~s}$ at $\left.95^{\circ} \mathrm{C}\right) . \mathrm{UBC} 18$ was used as an internal control. Gene expression was calculated using the $2^{\Delta \Delta \mathrm{Ct}}$ method. Primers used for gene expression analysis are listed in Supplementary Table 1.

Phylogenetic analysis. Phylogenetic trees were generated from the aligned protein sequences using MEGA6. Bootstrap test of phylogeny was performed using the neighbor-joining method from 1000 replications for each branch. Accession numbers for $B$. distachyon genes referred to in this study are as follows; BdFT1 (Bradilg48830), BdFT2 (Bradi2g07070), BdFTL10 (Bradi2g19670), BdFT4 (Bradilg38150), BdFTL9 (Bradi2g49795), BdFT6 (Bradi3g08890), BdFD1 (Bradi4g36587), BdVRN1 (Bradi3g08340), BdFUL2 (Bradilg59250), BdCO1 (Bradilg43670), BdCO2 (Bradi3g56260), BdVRN2 (Bradi3g10010).

Yeast two-hybrid assays. Full-length coding sequences of FT1, FTL9, FD1, and 14-3-3 were amplified from Bd21 B. distachyon cDNA by the primers listed in Supplementary Table 1. PCR products were cloned into the EcoRI and BamHI sites of the DNA-binding domain vector pGBKT7 or the GAL4-activation domain vector, pGADT7. Both vectors were co-transformed into yeast strain AH109 using the lithium acetate-based transformation protocol. The positive colonies were selected SD/-Leu/-Trp (-LT) medium and then used for a growth assay on selective $\mathrm{SD} /$-Leu/-Trp/-His/-Ade medium (-LTHA) supplemented with $1 \mathrm{mM} \mathrm{3-AT}$. The interactions were observed after 4 days of incubation at $30^{\circ} \mathrm{C}$. The quantitative interaction assays were performed using Chlorophenol red- $\beta$-D-galactopyranoside (Sigma) as substrates ${ }^{3}$. Yeast cells were broken by the freeze/thaw method and mixed with Chlorophenol red- $\beta$-D-galactopyranoside in a reaction Buffer. Reactions were stopped by adding $3 \mathrm{mM} \mathrm{ZnCl}$, and the color change of the reaction was measured at $578 \mathrm{~nm}$ using a UV/Vis spectrophotometer.

BiFC assays. Full-length coding sequences of the indicated proteins were constructed into vectors containing either N-terminal or C-terminal-enhanced yellow fluorescence protein fragments. Primers for the constructs are presented in Supplementary Table 1 . These resulting constructs were transformed into A. tumefaciens strain EHA105, which was then cultured overnight and adjusted to OD600 $=$ 0.8 . An equal volume of each culture was mixed together for injection. Sets of combination were co-infiltrated to 4 -week-old $N$. benthamiana leaves. YFP fluorescence was observed using a confocal laser scanning microscope (Zeiss, LSM700) 2-3 days later.

Luciferase imaging assays. For the firefly LUC complementation imaging assays, the full-length coding sequences of FD1 were ligated with $\mathrm{N}$-terminal fragment of luciferase (nLUC) to form FD1-nLUC. The full-length coding sequences of FT1 and FTL9 were fused with C-terminal fragment of luciferase (cLUC) to form cLUC-FT1 and cLUC-FTL9 to examine the interactions of FT1 and FTL9 with FD1. After a Luciferin $(100 \mu \mathrm{M})$ spray, the leaves were kept in a dark condition for $5 \mathrm{~min}$ before fluorescence observation. The images were taken by a low-light cooled charge-coupled device imaging apparatus (Tanon 5200).

To determine the flowering inductive efficiency of FTL9-FAC and FT1-FAC, equal concentrations and volumes of A. tumefaciens strains EHA105 harboring FD1 and the indicated FT or control proteins were mixed with $1 / 3$ amount of $A$. tumefaciens containing $3 \mathrm{~kb}$ VRN1 promoter-driven LUC constructs, and coinfiltrated into $N$. benthamiana leaves by a syringe. Similarly, equal concentrations and volumes of $A$. tumefaciens having $\mathrm{CO} 1$ and control proteins mixed with $1 / 3$ amount of those containing $3 \mathrm{~kb}$ FTL9 promoter-driven LUC constructs were cointroduced into $N$. benthamiana for a detection of the repressive activity of $\mathrm{CO} 1$ to FTL9. At least four leaves from independent $N$. benthamiana plants were infiltrated, and one leaf was for fluorescence signal observation and other leaves were punched and put in the $-80^{\circ} \mathrm{C}$ refrigerator to measure the LUC activity together later. For observation of the fluorescence signal, the injected tobacco leaves were kept in a dark condition for $5 \mathrm{~min}$ after a spray of Luciferin $(100 \mu \mathrm{M})$. The LUC images were taken by the imaging device (Tanon 5200). The LUC and REN activity of infiltrated leaf were measured via the Dual-Luciferase Reporter Assay System (Promega) on a GloMax 96 microplate luminometer (Promega), according to the manufacturer's instructions. Final transcriptional activity was indicated by the ratio of LUC/REN.
Reporting summary. Further information on experimental design is available in the Nature Research Reporting Summary linked to this article.

\section{Data availability}

The source data underlying Figs. 1-5 and Supplementary Figs. 1-3, 5-6, 8-11, and 13-14 are provided as a Source Data file. All other data that support the findings of this study are available from the corresponding author upon request.

Received: 31 August 2018 Revised: 24 January 2019 Accepted: 30 January 2019

Published online: 18 February 2019

\section{References}

1. Taoka, K. et al. 14-3-3 proteins act as intracellular receptors for rice Hd3a florigen. Nature 476, 332-335 (2011).

2. Li, C., Lin, H. \& Dubcovsky, J. Factorial combinations of protein interactions generate a multiplicity of florigen activation complexes in wheat and barley. Plant J. 84, 70-82 (2015).

3. Qin, Z. et al. Regulation of FT splicing by an endogenous cue in temperate grasses. Nat. Commun. 8, 14320 (2017).

4. Pin, P. A. et al. An antagonistic pair of FT homologs mediates the control of flowering time in sugar beet. Science 330, 1397-1400 (2010).

5. Song, Y. H., Shim, J. S., Kinmonth-Schultz, H. A. \& Imaizumi, T. Photoperiodic flowering: time measurement mechanisms in leaves. Annu. Rev. Plant Biol. 66, 441-464 (2015). 66.

6. Wenkel, S. et al. CONSTANS and the CCAAT box binding complex share a functionally important domain and interact to regulate flowering of Arabidopsis. Plant Cell 18, 2971-2984 (2006).

7. Gnesutta, N. et al. CONSTANS imparts DNA sequence specificity to the histone fold NF-YB/NF-YC dimer. Plant Cell 29, 1516-1532 (2017).

8. Tiwari, S. B. et al. The flowering time regulator CONSTANS is recruited to the FLOWERING LOCUS T promoter via a unique cis-element. New Phytol. 187, $57-66$ (2010).

9. Itoh, H., Nonoue, Y., Yano, M. \& Izawa, T. A pair of floral regulators sets critical day length for Hd3a florigen expression in rice. Nat. Genet 42, 635-638 (2010)

10. Andres, F. \& Coupland, G. The genetic basis of flowering responses to seasonal cues. Nat. Rev. Genet 13, 627-639 (2012).

11. $\mathrm{Wu}, \mathrm{L}$. et al. Regulation of FLOWERING LOCUS $\mathrm{T}$ by a microRNA in Brachypodium distachyon. Plant Cell 25, 4363-4377 (2013).

12. Qin, Z. R., Bai, Y. X. \& Wu, L. Flowering on time: multilayered restrictions on FT in plants. Mol. Plant 10, 1365-1367 (2017).

13. McKeown, M., Schubert, M., Preston, J. C. \& Fjellheim, S. Evolution of the miR5200-FLOWERING LOCUS T flowering time regulon in the temperate grass subfamily Pooideae. Mol. Phylogenet. Evol. 114, 111-121 (2017).

14. Hyun, Y. et al. Multi-layered regulation of SPL15 and cooperation with SOC1 integrate endogenous flowering pathways at the Arabidopsis shoot meristem. Dev. Cell 37, 254-266 (2016).

15. Boden, S. A. et al. EARLY FLOWERING3 regulates flowering in spring barley by mediating gibberellin production and FLOWERING LOCUS T expression. Plant Cell 26, 1557-1569 (2014).

16. Hill, C. B. \& Li, C. Genetic architecture of flowering phenology in cereals and opportunities for crop improvement. Front Plant Sci. 7, 1906 (2016).

17. Turner, A., Beales, J., Faure, S., Dunford, R. P. \& Laurie, D. A. The pseudoresponse regulator Ppd-H1 provides adaptation to photoperiod in barley. Science 310, 1031-1034 (2005).

18. Laurie, D. A., Pratchett, N., Snape, J. W. \& Bezant, J. H. RFLP mapping of five major genes and eight quantitative trait loci controlling flowering time in a winter x spring barley (Hordeum vulgare L.) cross. Genome 38, 575-585 (1995).

19. Halliwell, J. et al. Systematic investigation of FLOWERING LOCUS T-Like Poaceae gene families identifies the short-day expressed flowering pathway gene, TaFT3 in wheat (Triticum aestivum L.). Front Plant Sci. 7, 857 (2016).

20. Kikuchi, R., Kawahigashi, H., Ando, T., Tonooka, T. \& Handa, H. Molecular and functional characterization of PEBP genes in barley reveal the diversification of their roles in flowering. Plant Physiol. 149, 1341-1353 (2009).

21. Zikhali, M., Wingen, L. U., Leverington-Waite, M., Specel, S. \& Griffiths, S. The identification of new candidate genes Triticum aestivum FLOWERING LOCUS T3-B1 (TaFT3-B1) and TARGET OF EAT1 (TaTOE1-B1) controlling the short-day photoperiod response in bread wheat. Plant Cell Environ. 40, 2678-2690 (2017)

22. Faure, S., Higgins, J., Turner, A. \& Laurie, D. A. The FLOWERING LOCUS Tlike gene family in barley (Hordeum vulgare). Genetics 176, 599-609 (2007). 
23. Woods, D. P. et al. Establishment of a vernalization requirement in Brachypodium distachyon requires REPRESSOR OF VERNALIZATION1. Proc. Natl Acad. Sci. USA 114, 6623-6628 (2017)

24. Ream, T. S. et al. Interaction of photoperiod and vernalization determines flowering time of Brachypodium distachyon. Plant Physiol. 164, 694-709 (2014).

25. Lv, B. et al. Characterization of FLOWERING LOCUS T1 (FT1) gene in Brachypodium and wheat. PLoS ONE 9, e94171 (2014).

26. Woods, D. P., McKeown, M. A., Dong, Y. X., Preston, J. C. \& Amasino, R. M. Evolution of VRN2/Ghd7-like genes in vernalization-mediated repression of grass flowering. Plant Physiol. 170, 2124-2135 (2016).

27. Bettgenhaeuser, J. et al. Natural variation in Brachypodium links vernalization and flowering time loci as major flowering determinants. Plant Physiol. 173, 256-268 (2017).

28. Shaw, L. M., et al. FLOWERING LOCUS T2 (FT2) regulates spike development and fertility in temperate cereals. J. Exp. Bot. 70, 193-204 (2018).

29. Higgins, J. A., Bailey, P. C., Laurie, D. A. Comparative genomics of flowering time pathways using Brachypodium distachyon as a model for the temperate grasses. PLoS ONE 5, e10065 (2010).

30. Woods, D. P., Ream, T. S., Minevich, G., Hobert, O. \& Amasino, R. M. PHYTOCHROME C is an essential light receptor for photoperiodic flowering in the temperate grass, Brachypodium distachyon. Genetics 198, 397-408 (2014).

31. Chen, A. et al. Phytochrome $\mathrm{C}$ plays a major role in the acceleration of wheat flowering under long-day photoperiod. Proc. Natl Acad. Sci. USA 111, 10037-10044 (2014).

32. Pearce, S., Kippes, N., Chen, A., Debernardi, J. M. \& Dubcovsky, J. RNA-seq studies using wheat PHYTOCHROME B and PHYTOCHROME C mutants reveal shared and specific functions in the regulation of flowering and shadeavoidance pathways. BMC Plant Biol. 16, 141 (2016).

33. Li, C. \& Dubcovsky, J. Wheat FT protein regulates VRN1 transcription through interactions with FDL2. Plant J. 55, 543-554 (2008).

34. Mulki, M. A., Bi, X., von Korff M. FLOWERING LOCUS T3 controls spikelet initiation but not floral development. Plant Physiol. 178, 1170-1186 (2018).

35. Yamaguchi, A. et al. TWIN SISTER OFFT (TSF) acts as a floral pathway integrator redundantly with FT. Plant Cell Physiol. 48, S16-S16 (2007).

36. Mulki, M. A. \& von Korff, M. CONSTANS controls floral repression by upregulating VERNALIZATION2 (VRN-H2) in Barley. Plant Physiol. 170, 325-337 (2016).

37. Samach, A. et al. Distinct roles of CONSTANS target genes in reproductive development of Arabidopsis. Science 288, 1613-1616 (2000).

38. Lomax, A. et al. An ortholog of CURLY LEAF/ENHANCER OF ZESTE like-1 is required for proper flowering in Brachypodium distachyon. Plant J. 93 871-882 (2018).

39. Cockram, J., Horsnell, R., Soh, E. H., Norris, C., O'Sullivan, D. M. Molecular and phenotypic characterization of the alternative seasonal growth habit and flowering time in barley (Hordeum vulgare ssp vulgare L.). Mol. Breeding 35, 165 (2015).

40. Meng, X., Muszynski, M. G. \& Danilevskaya, O. N. The FT-like ZCN8 gene functions as a floral activator and is involved in photoperiod sensitivity in maize. Plant Cell 23, 942-960 (2011).

41. Lazakis, C. M., Coneva, V. \& Colasanti, J. ZCN8 encodes a potential orthologue of Arabidopsis FT florigen that integrates both endogenous and photoperiod flowering signals in maize. J. Exp. Bot. 62, 4833-4842 (2011).

42. Wolabu, T. W. et al. Three FLOWERING LOCUS T-like genes function as potential florigens and mediate photoperiod response in sorghum. New Phytol. 210, 946-959 (2016).

43. Wickland, D. P. \& Hanzawa, Y. The FLOWERING LOCUS T/TERMINAL FLOWER 1 gene family: functional Evolution and Molecular Mechanisms. Mol. Plant 8, 983-997 (2015).

44. Hayama, R., Yokoi, S., Tamaki, S., Yano, M. \& Shimamoto, K. Adaptation of photoperiodic control pathways produces short-day flowering in rice. Nature 422, 719-722 (2003)

45. Yano, M. et al. Hd1, a major photoperiod sensitivity quantitative trait locus in rice, is closely related to the arabidopsis flowering time gene CONSTANS. Plant Cell 12, 2473-2483 (2000).

46. Du, A. P. et al. The DTH8-Hd1 module mediates day-length-dependent regulation of rice flowering. Mol. Plant 10, 948-961 (2017).

47. Wei, X. J. et al. DTH8 suppresses flowering in rice, influencing plant height and yield potential simultaneously. Plant Physiol. 153, 1747-1758 (2010).

48. Hou, X. L., et al. Nuclear factor Y-mediated H3K27me3 demethylation of the SOC1 locus orchestrates flowering responses of Arabidopsis. Nat. Commun. 5, 4601 (2014).

49. Siriwardana, C. L., et al. NUCLEAR FACTOR Y, Subunit A (NF-YA) roteins positively regulate flowering and act through FLOWERING LOCUS T. PloS Genetics 12, e1006496 (2016).
50. Jang, S. et al. Arabidopsis COP1 shapes the temporal pattern of CO accumulation conferring a photoperiodic flowering response. EMBO J. 27, 1277-1288 (2008).

51. Liu, L. J. et al. COP1-mediated ubiquitination of CONSTANS is implicated in cryptochrome regulation of flowering in Arabidopsis. Plant Cell 20, 292-306 (2008).

52. Zuo, Z. C., Liu, H. T., Liu, B., Liu, X. M. \& Lin, C. T. Blue light-dependent interaction of CRY2 with SPA1 regulates COP1 activity and floral initiation in Arabidopsis. Curr. Biol. 21, 841-847 (2011).

53. Casao, M. C., et al. Adaptation of barley to mild winters: a role for PPDH2. BMC Plant Biol. 11, 164 (2011).

54. Casao, M. C. et al. Expression analysis of vernalization and day-length response genes in barley (Hordeum vulgare L.) indicates that VRNH2 is a repressor of PPDH2 (HvFT3) under long days. J. Exp. Bot. 62, 1939-1949 (2011).

55. Woods, D. P. et al. Genetic architecture of flowering-time variation in Brachypodium distachyon. Plant Physiol. 173, 269-279 (2017).

56. Ma, X. et al. A robust CRISPR/Cas9 system for convenient, high-efficiency multiplex genome editing in monocot and dicot plants. Mol. Plant 8, 1274-1284 (2015).

57. Alves, S. C. et al. A protocol for Agrobacterium-mediated transformation of Brachypodium distachyon community standard line Bd21. Nat. Protoc. 4, 638-649 (2009).

\section{Acknowledgements}

We sincerely thank Dr. Joyce Van Eck for helping us to establish B. distachyon transformation system. We also thank Dr. Yaoguang Liu and Richard M. Amasino for the gift of efficient CRISPR/Cas9 gene-editing system and B. distachyon phyc mutants, respectively. We appreciate Dr. Xueren Yin for assistance of LUC detection analysis. This work was supported by the National Natural Science Foundation of China (31570226, 91640109, 31700189), Zhejiang Provincial Natural Science Foundation of China (LR16C060001), China Postdoctoral Science Foundation (2017M610364, 2018T110586), the open funds of the State Key Laboratory of Crop Genetics and Germplasm Enhancement (ZW201807) and Hundred-Talent Program of Zhejiang University.

\section{Author contributions}

L.W. conceived and initiated the research. L.W. and Z.Q. designed the experiments. Z.Q. Y.B., S.M. and X.W. performed the experiments. Z.Q., Y.B., J.W., P.D., H.A. and L.W. analyzed the data. L.W. and Z.Q. wrote the article.

\section{Additional information}

Supplementary Information accompanies this paper at https://doi.org/10.1038/s41467019-08785-y.

Competing interests: The authors declare no competing interests.

Reprints and permission information is available online at http://npg.nature.com/ reprintsandpermissions/

Journal peer review information: Nature Communications thanks the anonymous reviewers for their contribution to the peer review of this work. Peer reviewer reports are available.

Publisher's note: Springer Nature remains neutral with regard to jurisdictional claims in published maps and institutional affiliations.

Open Access This article is licensed under a Creative Commons Attribution 4.0 International License, which permits use, sharing, adaptation, distribution and reproduction in any medium or format, as long as you give appropriate credit to the original author(s) and the source, provide a link to the Creative Commons license, and indicate if changes were made. The images or other third party material in this article are included in the article's Creative Commons license, unless indicated otherwise in a credit line to the material. If material is not included in the article's Creative Commons license and your intended use is not permitted by statutory regulation or exceeds the permitted use, you will need to obtain permission directly from the copyright holder. To view a copy of this license, visit http://creativecommons.org/ licenses/by/4.0/

(c) The Author(s) 2019 